\title{
Extensions of Automorphisms and Gauge Symmetries
}

\author{
Detlev Buchholz ${ }^{1}$, Sergio Doplicher ${ }^{2,}{ }^{\star}$, Roberto Longo ${ }^{3,}$, \\ and John E. Roberts ${ }^{3, \star}$ \\ 1 II. Institut für Theoretische Physik, Universität Hamburg, D-22761 Hamburg, Germany \\ 2 Dipartimento di Matematica, Università di Roma "La Sapienza", I-00185 Roma, Italy \\ 3 Dipartimento di Matematica, Università di Roma "Tor Vergata", I-00133 Roma, Italy
}

Received July 2, 1992

\section{Dedicated to Huzihiro Araki}

\begin{abstract}
We characterize the automorphisms of a $C^{*}$-algebra $\mathscr{A}$ which extend to automorphisms of the crossed product $\mathscr{B}$ of $\mathscr{A}$ by a compact group dual. The case where the inclusion $\mathscr{A} \subset \mathscr{B}$ is equipped with a group of automorphisms commuting with the dual action is also treated. These results are applied to the analysis of broken gauge symmetries in Quantum Field Theory to draw conclusions on the structure of the degenerate vacua on the field algebra.
\end{abstract}

\section{Introduction}

The first problem considered in this short note is that of extending automorphisms from a unital $C^{*}$-algebra $\mathscr{A}$ to the crossed product $\mathscr{B}$ by the action of a compact group dual. This problem appears, for example, in the structural analysis of symmetries in the algebraic setting of Quantum Field Theory. Having these applications in mind, we therefore work within the framework developed in [1], where the action of a compact group dual is determined by a specially directed symmetric monoidal subcategory $(\mathscr{T}, \varepsilon)$ of the category End $\mathscr{A}$ of unital endomorphisms of $\mathscr{A}$. We present necessary and sufficient conditions for an automorphism of $\mathscr{A}$ to be extendible to an automorphism of $\mathscr{B}$. These conditions are best summed up by considering the completed category $(\hat{\mathscr{T}}, \hat{\varepsilon})$ whose objects are the unital endomorphisms of $\mathscr{A}$ dominated by an object of $\mathscr{T}$. Then an automorphism $\beta$ of $\mathscr{A}$ extends to $\mathscr{B}$ if and only if it induces an automorphism of the symmetric monoidal category $(\hat{\mathscr{T}}, \hat{\varepsilon})$.

We also consider the problem of whether such automorphisms can be chosen to commute with a given group $T$ of automorphisms of the crossed product $\mathscr{B}$ which leaves $\mathscr{A}$ invariant as a set and commutes with the dual action.

As an immediate application, we consider an infinite factor acted on by a compact group where the fixed-point algebra has trivial relative commutant

\footnotetext{
* Research supported by MURST and CNR, GNAFA
} 
and characterize the automorphisms of the fixed-point algebra which extend to an automorphism of the original factor (Sect. 2).

Turning to the applications to Quantum Field Theory, it was shown in [2] that there is a canonical field net associated with a net of local observables. In the absence of spontaneously broken symmetries, this field net is basically given as the crossed product of the net of local observables by the action of the dual of the compact gauge group. In this context, $\mathscr{T}$ describes the superselection structure associated with localizable charges and $T$ is the group of translations in spacetime. Our present results allow one to extend local automorphisms of $\mathfrak{U}$ to local automorphisms of the field net $\mathfrak{F}$, possibly commuting with translations (Sect. 3).

In Sect. 4 we apply these results to study the spontaneous breakdown of gauge symmetry in Quantum Field Theory in the framework described in [3]. This phenomenon can occur when essential duality but not duality holds in the vacuum sector. The full group $\Gamma$ of gauge symmetries is the (pointwise) stabilizer of $\mathfrak{U}$ in $\mathfrak{F}$ and the elements of $\Gamma$ which do not leave the vacuum state on $\mathfrak{F}$ invariant are the spontaneously broken symmetries. The subgroup $G$ leaving the vacuum state invariant consists precisely of those symmetries which also leave the dual net $\mathfrak{I}^{d}$ pointwise invariant. A spontaneously broken symmetry maps the vacuum state on $\mathfrak{F}$ into another vacuum state which is also an extension of the vacuum state on $\mathfrak{A}$. We pose the question of whether all such extensions arise from broken symmetries but give only a partial answer characterizing the $G$-invariant extensions which preserve essential duality.

This characterization applies whenever $\Gamma$ commutes with translations as it does if there are local energy-momentum operators associated with the observable algebra [3]. Should $\Gamma$ not commute with translations then we still obtain a simple variant of our characterization when the centre of $G$ is discrete.

\section{Extensions of Automorphisms}

Let $\mathscr{A}$ be a $C^{*}$-algebra with centre $\mathbb{C} I,(\Delta, \varepsilon)$ a permutation symmetric, specially directed semigroup of unital endomorphisms of $\mathscr{A}$. In other words, cf. [1], if we consider the full subcategory $\mathscr{T}$ of End $\mathscr{A}$ with objects $\Delta$ then $(\mathscr{T}, \varepsilon)$ is a symmetric monoidal category. Furthermore, $\mathscr{T}$ has sufficient special objects, i.e. objects with determinant one. Such an object $\varrho$ is characterized by there being an isometry $R \in\left(l, \varrho^{d}\right)$ with

$$
\begin{gathered}
R^{*} \varrho(R)=(-1)^{d-1} d^{-1} I, \\
R R^{*}=(d !)^{-1} \sum_{p \in \mathbb{P}_{d}} \operatorname{sign}(p) \varepsilon_{\varrho}(p),
\end{gathered}
$$

where $d$ denotes the dimension of $\varrho$ and $\varepsilon_{\varrho}$ the unitary representation of the group $\mathbb{P}_{\infty}$ of finite permutations of the integers derived from $\varepsilon . \mathbb{P}_{d}$ is considered in the obvious fashion as a subgroup of $\mathbb{P}_{\infty}$. As is shown in [1], these conditions mean that $(\mathscr{T}, \varepsilon)$ is an abstract dual of a unique compact group $G$ which is the stabilizer of $\mathscr{A}$ in a crossed product $\mathscr{B}$ of $\mathscr{A}$ by $\mathscr{T}$ :

$$
\mathscr{B}=\mathscr{A} \times \mathscr{T} ; \quad \mathscr{A}=\mathscr{B}^{G}
$$

and we have

$$
\mathscr{A}^{\prime} \cap \mathscr{B}=\mathbb{C} I .
$$


$\mathscr{B}$ is generated by $\mathscr{A}$ and the subsets

$$
H_{\varrho}:=\{\psi \in \mathscr{B}: \psi A=\varrho(A) \psi, A \in \mathscr{A}\}
$$

for $\varrho \in \Delta$. With the norm induced by $\mathscr{B}, H_{\varrho}$ is a Hilbert space which has support $I$, i.e. if $\psi_{i}$ is an orthonormal basis then

$$
\sum_{i} \psi_{i} \psi_{i}^{*}=I
$$

Any finite-dimensional Hilbert space $H$ in a $C^{*}$-algebra $\mathscr{B}$ induces a canonical endomorphism $\sigma_{H}$ defined by

$$
\sigma_{H}(B):=\sum_{i} \psi_{i} B \psi_{i}^{*}
$$

If $H$ and $K$ are two Hilbert spaces in $\mathscr{B}$, then $\theta(H, K)$ denotes the canonical unitary operator in $\mathscr{B}$ permuting the tensor product of the two spaces

$$
\theta(H, K) \phi \psi=\psi \phi, \quad \phi \in H, \quad \psi \in K .
$$

The problem we consider here is whether an automorphism of $\mathscr{A}$ can be extended to an automorphism of $\mathscr{B}$ and we begin by considering automorphisms $\beta$ of $\mathscr{A}$ which normalize $\Delta$, i.e.

$$
\beta \Delta \beta^{-1}=\Delta
$$

In this case, necessary and sufficient conditions for $\beta$ to extend to $\mathscr{B}$ are given in

Proposition 2.1. An automorphism $\beta$ of $\mathscr{A}$ which normalizes $\Delta$ extends to an automorphism $\widetilde{\beta}$ of $\mathscr{B}$ if and only if

$$
\beta\left(\varepsilon\left(\varrho, \varrho^{\prime}\right)\right)=\varepsilon\left(\beta \varrho \beta^{-1}, \beta \varrho^{\prime} \beta^{-1}\right), \quad \varrho, \varrho^{\prime} \in \Delta .
$$

If, furthermore, $\beta$ is unitarily implemented in a faithful representation $\pi_{0}$ of $\mathscr{A}$, $\widetilde{\beta}$ is unitarily implemented in the representation $\pi$ of $\mathscr{B}$ obtained by inducing up from $\pi_{0}$.

Proof. The first assertions are immediate consequences of the uniqueness statements in [1, Theorem 5.1, Theorem 6.1]. Letting $\eta$ denote the inclusion mapping of $\mathscr{A}$ into $\mathscr{B}$, uniqueness says that, given two systems as in [1, Theorem 5.1], say $\left\{\mathscr{B}, \eta, G, \varrho \in \Delta \mapsto H_{\varrho}\right\}$ and $\left\{\mathscr{B}_{1}, \eta_{1}, G_{1}, \varrho \in \Delta \mapsto H_{\varrho}^{1}\right\}$, there is a $*$-isomorphism $\varphi$ of $\mathscr{B}$ onto $\mathscr{B}_{1}$ such that

$$
\varphi \circ \eta=\eta_{1} ; \quad \varphi\left(H_{\varrho}\right)=H_{\varrho}^{1}, \quad \varrho \in \Delta .
$$

Furthermore, if we let $\mathscr{B}, \mathscr{B}_{1}$ be the spatial versions of the crossed product as in [1, Theorem 6.1], and $\pi, \pi_{1}$ be the associated representations of $\mathscr{A}$ on the Hilbert spaces $\mathscr{H}, \mathscr{H}_{1}$, reducing to the faithful representations $\pi_{0}$ and $\pi_{01}$ on the subspaces $\mathscr{H}_{0}, \mathscr{H}_{01}$ of $G$-invariant and $G_{1}$-invariant vectors, respectively, then, a unitary operator $U$ from $\mathscr{H}_{0}$ to $\mathscr{H}_{01}$ such that

$$
U \pi_{0}(A)=\pi_{01}(A) U, \quad A \in \mathscr{A},
$$

will extend to a unitary $W$ of $\mathscr{H}$ onto $\mathscr{H}_{1}$ by setting (cf. the proof of [1, Theorem 6.1])

$$
W \sum_{i} B_{i} \Phi_{i}=\sum_{i} \varphi\left(B_{i}\right) U \Phi_{i}, \quad B_{i} \in \mathscr{B}, \quad \Phi_{i} \in \mathscr{H}_{0}
$$


and this unitary will satisfy

$$
\begin{array}{cc}
W \pi(A)=\pi_{1}(A) W, & A \in \mathfrak{U} ; \\
W B W^{-1}=\varphi(B), & B \in \mathscr{B} ; \\
W \mid \mathscr{H}_{0}=U . &
\end{array}
$$

Now given a system $\left\{\mathscr{B} ; \eta ; G, \varrho \in \Delta \mapsto H_{\varrho}\right\}$ as in [1, Theorem 5.1], Eqs. (2.1), (2.2) allow us to define another such system $\left\{\mathscr{B}, \eta \circ \beta, G, \varrho \in \Delta \mapsto H_{\beta \varrho \beta^{-1}}\right\}$. Hence, in this case, the map $\varphi$ is an automorphism $\widetilde{\beta}$ of $\mathscr{B}$ such that

$$
\begin{gathered}
\widetilde{\beta}(A)=\beta(A), \quad A \in \mathscr{A} ; \\
\widehat{\beta}\left(H_{\varrho}\right)=H_{\beta \varrho \beta^{-1}}, \quad \varrho \in \Delta .
\end{gathered}
$$

If $\pi_{0}$ is a faithful representation of $\mathscr{A}$ on $\mathscr{H}_{0}$ and $U$ is a unitary operator on $\mathscr{H}_{0}$ such that

$$
U \pi_{0}(A) U^{-1}=\pi_{0} \circ \beta(A), \quad A \in \mathfrak{U},
$$

then, in view of the preceding remarks, if we consider the representations of $\mathscr{B}$ induced up from $\pi_{0}$ and $\pi_{01}:=\pi_{0} \circ \beta$, we see that the automorphism $\widetilde{\beta}$ of $\mathscr{B}$ is implemented by $W$ in the induced representation. Conversely, if $\widetilde{\beta}$ is an automorphism of $\mathscr{B}$ extending $\beta$, Eq. (2.5) follows from

$$
H_{\varrho}=\{\psi \in \mathscr{B} \mid \psi A=\varrho(A) \psi, A \in \mathscr{A}\}
$$

and Eq. (2.2) follows from

$$
\varepsilon\left(\varrho, \varrho^{\prime}\right)=\theta\left(H_{\varrho}, H_{\varrho^{\prime}}\right), \quad \varrho, \varrho^{\prime} \in \Delta .
$$

Now the condition that our automorphism $\beta$ should normalize $\Delta$,

$$
\beta \Delta \beta^{-1}=\Delta,
$$

is unnecessarily restrictive and can be easily modified to yield necessary and sufficient conditions for an automorphism $\beta$ of $\mathscr{A}$ to extend to $\mathscr{B}$. The point is that $\Delta$ and hence $\mathscr{T}$ is not uniquely determined in the sense that there are other choices yielding the same crossed product. However, there is a maximal choice $\hat{\Delta} \supset \Delta ; \hat{\Delta}$ is the set of unital endomorphisms of $\mathscr{A}$ induced by finite dimensional Hilbert spaces in $\mathscr{B}$. lemma.

An equivalent characterization of $\hat{\Delta}$ is given by the following elementary

Lemma 2.2. Let $\mathscr{B}$ be a $C^{*}$-algebra with unit and $G$ a group of automorphisms such that $\mathscr{A}^{\prime} \cap \mathscr{B}=\mathbb{C} \cdot I$, with $\mathscr{A}$ the $G$-fixed points in $\mathscr{B}$. A finite-dimensional Hilbert space $H$ with support I in $\mathscr{B}$ induces an endomorphism @ of $\mathscr{A}$ if and only if it is G-stable.

Proof. If $H$ induces an endomorphism $\varrho$ of $\mathscr{A}$ we have, for each $\psi \in H$,

$$
\psi A=\varrho(A) \psi, \quad A \in \mathscr{A} .
$$

Since $\mathscr{A}^{\prime} \cap \mathscr{B}=\mathbb{C} \cdot I$, the set of all $\psi \in \mathscr{B}$ fulfilling this equation is a Hilbert space which is obviously $G$-stable; it includes $H$ and hence coincides with $H$ because $H$ has support $I$. If $H$ is $G$-stable, the inner endomorphism $\varrho_{H}$ of $\mathscr{B}$ commutes with $G$ and hence leaves $\mathscr{A}$ globallx stable. 
Remark. More generally, finite-dimensional Hilbert spaces $H$ with support $1_{H}$ in $\mathscr{B}$ induce endomorphisms $\varrho$ of $\mathscr{A}$ with $\varrho(I)=1_{H}$, if and only if they are $G$-invariant.

Now let $\hat{\mathscr{T}}$ denote the full subcategory of End $\mathscr{A}$ with objects $\hat{\Delta}$. This is the full subcategory of the symmetric monoidal category $\mathscr{S}(\mathscr{B}, \alpha)$ considered in [4] determined by the objects which are unital endomorphisms. The symmetry $\hat{\varepsilon}$ is determined by

$$
\hat{\varepsilon}(\varrho, \sigma)=\theta(H, K),
$$

where $H$ and $K$ are the Hilbert spaces inducing $\varrho$ and $\sigma$, respectively. $(\hat{\mathscr{T}}, \hat{\varepsilon})$ is therefore a symmetric monoidal category and $\hat{\varepsilon}$ extends $\varepsilon$. It can be easily checked that $\hat{\Delta}$ satisfies the conditions imposed on $\Delta$ above. Furthermore, by the uniqueness of the crossed product [1, Theorem 5.1], the algebra $\mathscr{B}$ is the crossed product of $\mathscr{A}$ by $\hat{\mathscr{T}}$ so that

$$
\mathscr{A} \times \mathscr{T}=\mathscr{A} \times \hat{\mathscr{T}} .
$$

Moreover, if $\widetilde{\beta}$ is an automorphism of the inclusion $\mathscr{A} \subset \mathscr{B}$, i.e. if $\widetilde{\beta}$ is an automorphism of $\mathscr{B}$ such that $\widetilde{\beta}(\mathscr{A})=\mathscr{A}$, then clearly

$$
\widetilde{\beta} \hat{\Delta} \widetilde{\beta}^{-1}=\hat{\Delta} .
$$

$\hat{\Delta}$ may be alternatively described as the set of objects in End $\mathscr{A}$ which are direct sums of subobjects of elements of $\Delta[4$, Lemma 6.2] or as the set of objects which are dominated by an object of $\Delta$.

We are now led to the following

Proposition 2.3. An automorphism $\beta$ of $\mathscr{A}$ extends to an automorphism $\widetilde{\beta}$ of $\mathscr{B}$ if and only if

a) $\beta \Delta \beta^{-1} C \hat{\Delta}, \beta^{-1} \Delta \beta C \hat{\Delta}$;

b) $\beta(\varepsilon(\varrho, \sigma))=\hat{\varepsilon}\left(\beta \varrho \beta^{-1}, \beta \sigma \beta^{-1}\right), \varrho, \sigma \in \Delta$.

Proof. Obviously, a) implies $\beta \widehat{\Delta} \beta^{-1} \subset \hat{\Delta}$ and the reverse inclusion whereas b) also holds for $\varrho, \sigma \in \hat{\Delta}$ as follows easily from the basic property of a symmetry and the fact that each element of $\hat{\Delta}$ is a direct sum of subobjects of elements of $\Delta$.

An alternatively way of looking at this result is to say that $\beta$ extends to an automorphism of $\mathscr{B}$ if and only if it induces an automorphism of the symmetric monoidal category $(\hat{\mathscr{T}}, \hat{\varepsilon})$.

Corollary 2.4. Let $N \subset M$ be an inclusion of infinite factors such that $N^{\prime} \cap M=\mathbb{C} \cdot I$ and $G$ a compact group of automorphisms of $M$ such that $N=M^{G}$. Let $(\mathscr{T}, \varepsilon)$ be the full symmetric monoidal subcategory of $\mathscr{S}(M, \alpha)$ determined by the objects which are unital endomorphisms, where $\varepsilon(\varrho, \sigma)=\theta(H, K)$ if $\varrho, \sigma$ are induced by the Hilbert spaces $H, K$, respectively, and $\alpha$ is just the inclusion into Aut $M$. An automorphism of $N$ extends to an automorphism of $M$ if and only if it induces an automorphism of $(\mathscr{T}, \varepsilon)$ as a symmetric monoidal category.

Proof. By [1, Sect. 7] $M$ arises from $N$ as the $W^{*}$-crossed product of $N$ by a compact group dual $(\mathscr{T}, \varepsilon)$, and $G$ is the dual action. The result follows by combining the argument in [1] with the preceding propositions. 
A related result on extending automorphisms of a von Neumann algebra to the crossed product by modular groups can be found in [5, Proposition 12.1]. This, too, relies on the uniqueness of the crossed product [6].

Now suppose we are given an action $\alpha$ of $T$ on $\mathscr{A}$ extending to an action $\tilde{\alpha}$ of $T$ on $\mathscr{B}$ commuting with $G$. As we know from [1, Sect. 8] such an extension exists if and only if there is a unitary cocycle $W_{\varrho}(t) \in\left(\varrho, \alpha_{t} \varrho \alpha_{t}^{-1}\right), \varrho \in \Delta, t \in T$ determined by

$$
\tilde{\alpha}_{t}(\psi)=W_{\varrho}(t) \psi, \quad \psi \in H_{\varrho}, \quad \varrho \in \Delta
$$

and satisfying

$$
\begin{gathered}
W_{\varrho \varrho^{\prime}}(t)=W_{\varrho}(t) \varrho\left(W_{\varrho^{\prime}}(t)\right), \quad \varrho, \varrho^{\prime} \in \Delta, \quad t \in T, \\
W_{\varrho^{\prime}}(t) S W_{\varrho}(t)^{*}=\alpha_{t}(S), \quad S \in\left(\varrho, \varrho^{\prime}\right), \quad \varrho, \varrho^{\prime} \in \Delta, \\
W_{\varrho}\left(t t^{\prime}\right)=\alpha_{t}\left(W_{\varrho}\left(t^{\prime}\right)\right) W_{\varrho}(t) .
\end{gathered}
$$

Now let $\beta$ be an automorphism of $\mathscr{A}$ and $\widetilde{\beta}$ an extension of $\beta$ to an automorphism of $\mathscr{B}$. Without loss of generality, we may suppose that $\beta$ normalizes $\Delta$. Then if

$$
\begin{aligned}
& \beta \alpha_{t}=\alpha_{t} \beta, \quad t \in T, \\
& \beta\left(W_{\varrho}(t)\right)=W_{\beta \varrho \beta^{-1}}(t)
\end{aligned}
$$

hold, we have for each $\varrho \in \Delta$ and $\psi \in H_{\varrho}$,

$$
\begin{gathered}
\widetilde{\beta} \tilde{\alpha}_{t}(\psi)=\widetilde{\beta}\left(W_{\varrho}(t) \psi\right)=\beta\left(W_{\varrho}(t)\right) \widetilde{\beta}(\psi), \\
\tilde{\alpha}_{t} \tilde{\beta}(\psi)=W_{\beta \varrho \beta^{-1}}(t) \widetilde{\beta}(\psi) ;
\end{gathered}
$$

so that $\tilde{\beta} \tilde{\alpha}_{t}=\tilde{\alpha}_{t} \tilde{\beta}$ both on $\mathscr{A}$ and on each $H_{\varrho}, \varrho \in \Delta$. Since these sets generate $\mathscr{B}$, $\widetilde{\beta}$ and $\tilde{\alpha}_{t}$ commute. Conversely, if $\tilde{\beta}$ and $\tilde{\alpha}_{t}$ commute for $t \in T,(2.8)$ and (2.9) show that (2.7) holds, too.

We next study the situation where $\beta$ fulfills (2.1), (2.2), (2.6) but (2.7) is not required a priori. In our application in Sect. $4, T$ will be the group of translations in spacetime, so we assume here that $T$ is a connected topological group and that $t \in T \mapsto \tilde{\alpha}_{t}(B)$ is strong-operator continuous for each $B \in \mathscr{B}$ in a spatial version of the crossed product.

We let $H_{0} \subset$ Aut $\mathscr{A}$ denote the group of all $\beta \in$ Aut $\mathscr{A}$ satisfying (2.1), (2.2), and (2.6) and note first that $H_{0}$ acts on the centre $Z(G)$ of $G$ as follows: if $\widetilde{\beta}$ is the extension of $\beta \in H_{0}$ to $\mathscr{B}, \widetilde{\beta}$ is unique up to an element of $G$ and normalizes $G$ (since $\widetilde{\beta} g \widetilde{\beta}^{-1}$ leaves $\mathscr{A}$ pointwise fixed for each $g \in G$ and is thus an element of $G$ ). Hence $\widetilde{\beta}$ induces an automorphism

$$
z \in Z(G) \mapsto{ }^{\beta} z:=\widetilde{\beta} z \widetilde{\beta}^{-1} \in Z(G)
$$

of $Z(G)$ which is independent of the choice of extension $\widetilde{\beta}$ of $\beta$.

If $\operatorname{Hom}\left(T, Z_{0}(G)\right)$ denotes the set of continuous homomorphisms of $T$ into $Z(G)$ [i.e. into the connected component $Z_{0}(G)$ of the identity in $Z(G)$ since $T$ is connected], we have an action of $H_{0}$ on $\operatorname{Hom}\left(T, Z_{0}(G)\right)$ defined by

$$
\left({ }^{\beta} z\right)(t)={ }^{\beta} z(t), \quad t \in T, \quad z \in \operatorname{Hom}\left(T, Z_{0}(G)\right),
$$

and we consider 1-cocycles of $H_{0}$ with values in $\operatorname{Hom}\left(T, Z_{0}(G)\right)$ for the action (2.10). 
Proposition 2.5. Given $\{\mathscr{A},(\Delta ; \varepsilon), \alpha\}$ as above, there is a well defined 1-cocycle $z$ on $H_{0}$ with values in $\operatorname{Hom}\left(T, Z_{0}(G)\right)$ such that $z_{\beta}$ is the identity if and only if $\beta$ extends to an automorphism of $\mathscr{B}$ commuting with $\tilde{\alpha}_{t}, t \in T$, i.e. if and only if $\beta$ satisfies (2.7). In particular, this will be the case for each $\beta \in H_{0}$ if $G$ has discrete centre.

Proof. Let $\widetilde{\beta}$ be an automorphism of $\mathscr{B}$ extending $\beta \in H_{0}$. We already saw that $\widetilde{\beta}$ is in the normalizer of $G$ and since $\tilde{\alpha}_{t}$ is in the centralizer of $G$, the commutator $\widetilde{\beta} \tilde{\alpha}_{t} \widetilde{\beta}^{-1} \tilde{\alpha}_{t}^{-1}$, which is independent of the choice of the extension $\widetilde{\beta}$ of $\beta$, commutes with the elements of $G$. But it also leaves $\mathscr{A}$ pointwise fixed since $\beta$ and $\alpha_{t}$ commute, hence it is also in $G$ and is easily checked to depend multiplicatively on $t$. Hence it defines a continuous homomorphism of $T$ into $Z(G)$ which takes values in $Z_{0}(G)$ since $T$ is connected:

$$
\tilde{\beta} \tilde{\alpha}_{t} \widetilde{\beta}^{-1} \tilde{\alpha}_{t}^{-1}=z_{\beta}(t) \in Z_{0}(G) .
$$

Now if $\beta_{1}, \beta_{2} \in H_{0},\left(\beta_{1} \beta_{2}\right)^{\sim}=\widetilde{\beta}_{1} \widetilde{\beta}_{2} g_{0}$ for some $g_{0} \in G$ and for given extensions of $\beta_{1}, \beta_{2}, \beta_{1} \beta_{2}$ to automorphisms of $\mathscr{B}$. Hence

$$
\begin{aligned}
z_{\beta_{1} \beta_{2}}(t) \tilde{\alpha}_{t} & =\left(\beta_{1} \beta_{2}\right)^{2} \tilde{\alpha}_{t}\left(\beta_{1} \beta_{2}\right)^{-1}=\widetilde{\beta}_{1} \widetilde{\beta}_{2} g_{0} \tilde{\alpha}_{t} g_{0}^{-1} \widetilde{\beta}_{2}^{-1} \widetilde{\beta}_{1}^{-1}=\widetilde{\beta}_{1} \widetilde{\beta}_{2} \tilde{\alpha}_{t} \widetilde{\beta}_{2}^{-1} \widetilde{\beta}_{1}^{-1} \\
& =\widetilde{\beta}_{1} z_{\beta_{2}}(t) \tilde{\alpha}_{t} \widetilde{\beta}_{1}^{-1}={ }^{\beta_{1}} z_{\beta_{2}}(t) z_{\beta_{1}}(t) \tilde{\alpha}_{t}
\end{aligned}
$$

so that

$$
z_{\beta_{1} \beta_{2}}=z_{\beta_{1}}^{\beta_{1}} z_{\beta_{2}}
$$

and $z$ is the anticipated 1-cocycle. Obviously, by (2.11), $z_{\beta}=1$ if and only if one (and hence any) extension of $\beta$ to an automorphism of $\mathscr{B}$ commutes with $\tilde{\alpha}_{t}$, $t \in T$.

\section{An Exact Sequence of Symmetries}

We now turn to some applications of the preceding results to Quantum Field Theory. We consider a net $\mathfrak{A}$ of local observables satisfying duality in the vacuum sector, i.e.

$$
\mathfrak{A}(\mathcal{O})=\mathfrak{U}^{d}(\mathcal{O}):=\mathfrak{A}\left(\mathcal{O}^{\prime}\right)^{\prime},
$$

for each double cone $\mathcal{O}$. This means that, for the moment, we suppose that there are no spontaneously broken gauge symmetries. Under these circumstances, there is a canonical field net $\mathfrak{F}$ with Bose-Fermi commutation relations containing $\mathfrak{A}$ and $\mathfrak{A}$ is the fixed-point net of $\mathfrak{F}$ under the action of a compact group $G$ which is the group of all automorphisms of $\mathfrak{F}$ leaving $\mathfrak{A}$ pointwise fixed.

From a physical point of view, the most obvious question concerning the extension of automorphisms from $\mathfrak{A}$ to $\mathfrak{F}$ is that of the extension of spacetime symmetries. In this case, the extensions commute with gauge symmetries and the underlying mathematical theory was already treated in $[1$, Sect. 8] and the application to Quantum Field Theory was treated in detail in [2, Sect. 6]. For the problem to have a positive solution it is necessary to restrict oneself to the subsemigroup $\Delta_{c}$ of $\Delta$, the semigroup of localized morphisms with finite statistics, consisting of covariant morphisms. However, under mild regularity conditions it has been recently shown that each element of $\Delta$ is in fact covariant [7]. 
Another related problem concerns the extension of KMS states from $\mathfrak{A}$ to $\mathfrak{F}$ and hence of the extension of the associated KMS automorphisms. This, too, has already been treated in the literature [8].

We now turn to a third problem, that of extending local symmetries of the theory and we first give a discussion without assuming that the theory is translation covariant. The net $\mathfrak{A}$ is merely required to be irreducible and to satisfy Property B (cf. [2]).

Let $H_{l}$ denote the group of all symmetries of the net $\mathfrak{A}$, i.e. $\beta \in H_{l}$ if and only if $\beta \in$ Aut $\mathfrak{A}$ and $\beta(\mathfrak{U}(\mathcal{O}))=\mathfrak{U}(\mathcal{O}), \mathcal{O} \in \mathscr{K}$. Similarly, we let $K_{l}$ denote the symmetries of the pair $(\mathfrak{U}, \mathfrak{F})$, i.e. the group of all symmetries of the net $\mathfrak{F}$ which leave the net $\mathfrak{U}$ globally stable; hence $G \subset K_{l}$ and if $\widetilde{\beta} \in K_{l}$ then its restriction to $\mathfrak{A}$ belongs to $H_{l}$. We now have

Proposition 3.1. There is a short exact sequence

$$
1 \rightarrow G \rightarrow K_{l} \rightarrow H_{l} \rightarrow 1,
$$

where the second and third arrows are just inclusion and restriction to $\mathfrak{U}$, respectively.

Proof. The kernel of the restriction of $K_{l}$ to $\mathfrak{U}$ is precisely $G$, so that we have only to prove that each $\beta \in H_{l}$ extends to an element $\widetilde{\beta}$ of $K_{l}$. This is a direct consequence of the comments following [2, Theorem 3.6] but we give here an explicit proof drawing on the results of Sect. 2. For each double cone $\mathcal{O}$, $\mathfrak{F}(\mathcal{O})$ can be thought of as the $W^{*}$-form of the crossed product of $\mathfrak{U}(\mathcal{O})$ by $\mathscr{T}_{\mathscr{O}}$, the full subcategory of End $\mathfrak{U}(\mathcal{O})$ with objects $\Delta(\mathcal{O})=\{\varrho \mid \mathfrak{U}(\mathcal{O}): \varrho \in \Delta, \varrho(A)=A$, $\left.A \in \mathfrak{U}\left(\mathcal{O}^{\prime}\right)\right\}$ and whose symmetry is the Bosonized symmetry $\hat{\varepsilon}$ ([2, Sect. 3$]$ and [1. Sect. 7]). We now verify the validity of relations (2.1) and (2.2). If $\varrho$ is a transportable localized morphism localized in $\mathcal{O}$ and $\beta \in H_{l}$ then so is $\beta \varrho \beta^{-1}$ since $\beta$ acts locally. Now consider the permutation symmetry which describes statistics:

$$
\varepsilon\left(\varrho, \varrho^{\prime}\right)=\left(U^{\prime} \times U\right) * U \times U^{\prime},
$$

where $U \in\left(\varrho, \varrho_{1}\right)$ and $U^{\prime} \in\left(\varrho^{\prime}, \varrho_{1}^{\prime}\right)$ are any unitary intertwiners such that $\varrho_{1}$ and $\varrho_{1}^{\prime}$ are localized spacelike to one another. Here $x$ and $\circ$ denote the monoidal and composition product in our monoidal category (cf. [9]). Since $\beta$ acts locally, $\beta \varrho_{1} \beta^{-1}$ and $\beta Q_{1}^{\prime} \beta^{-1}$ are localized spacelike to one another and

$$
\beta(U) \in\left(\beta \varrho \beta^{-1}, \beta \varrho_{1} \beta^{-1}\right), \quad \beta\left(U^{\prime}\right) \in\left(\beta \varrho^{\prime} \beta^{-1}, \beta \varrho_{1}^{\prime} \beta^{-1}\right)
$$

so that

$$
\beta\left(\varepsilon\left(\varrho, \varrho^{\prime}\right)\right)=\left(\beta\left(U^{\prime}\right) \times \beta(U)\right) * \circ \beta(U) \times \beta\left(U^{\prime}\right)=\varepsilon\left(\beta \varrho \beta^{-1}, \beta \varrho^{\prime} \beta^{-1}\right) .
$$

Now if $\varphi$ is a left inverse for $\varrho$ then $\beta \varphi \beta^{-1}$ is a left inverse for $\beta \varrho \beta^{-1}$ and if $\varphi$ is a standard left inverse then so is $\beta \varphi \beta^{-1}$ (this terminology was introduced in [9]). Hence if $\varrho$ has finite statistics then so does $\beta \varrho \beta^{-1}$ and (2.1) is satisfied. Simple computations (cf. Eq. (1.9), (7.3), (7.16), and (7.17) of [10]) then show that

$$
\beta\left(\hat{\varepsilon}\left(\varrho, \varrho^{\prime}\right)\right)=\hat{\varepsilon}\left(\beta \varrho \beta^{-1}, \beta \varrho^{\prime} \beta^{-1}\right)
$$

Thus $\beta$ extends to an automorphism of the $C^{*}$-crossed product. Since $\beta$ induces an automorphism of each $\mathfrak{A}(\mathcal{O})$, a von Neumann algebra on a separable 
Hilbert space with a properly infinite commutant (by Property B and local commutativity), $\beta$ is locally unitarily implemented and so is its extension to the crossed product by Proposition 2.1 . Thus $\beta$ extends locally to the $W^{*}$-closures $\mathfrak{F}(\mathcal{O})$ and hence globally to an automorphism $\widetilde{\beta}$ of $\mathfrak{F}$ which belongs to $K_{l}$ and extends $\beta$.

We now consider a translation covariant theory where the vacuum sector fulfills the spectrum condition. Denote by $H_{l}^{c}$ the elements of $H_{l}$ which commute with the action $\alpha$ and by $K_{l}^{c}$ the automorphisms of the net $\mathfrak{F}$ which commute with the action $\tilde{\alpha}$ of translations and leave the net $\mathfrak{A}$ globally stable, where $\mathfrak{F}$ now refers to the covariant complete normal field system with gauge symmetry (cf. [2, Sect. 6]). Since $\beta \in H_{l}^{c}$ commutes with $\alpha$ we have $\beta \Delta_{c} \beta^{-1}=\Delta_{c}$ and, reasoning as above using Proposition 2.5, we can deduce the following result.

Corollary 3.2. If the gauge group $G$ has a discrete centre, there is a short exact sequence

$$
1 \rightarrow G \rightarrow K_{l}^{c} \rightarrow H_{l}^{c} \rightarrow 1,
$$

where the second arrow is inclusion and the third one is the restriction to $\mathfrak{A}$.

Corollary 3.2 will be applied in the next section to study vacuum degeneracy in the case of spontaneously broken gauge symmetry. We do not know whether the corollary might not hold without any assumption on the centre of $G$ as a consequence of the spectrum condition which has not been directly exploited here.

\section{Vacuum Degeneracy and Gauge Automorphisms}

We now apply the results derived in the previous section to study the problem of vacuum degeneracy in theories with spontaneously broken gauge symmetries. The setting here is that described in [3] and we begin with a net $\mathfrak{U}$ of observables satisfying essential duality

$$
\mathfrak{U}^{d}=\mathfrak{U}^{d d}
$$

The canonical field net $\mathfrak{F}$ is now given by the construction recalled in the previous section but starting with $\mathfrak{\mathfrak { A }}^{d}$ rather than $\mathfrak{A}$ so that we now have inclusions

$$
\mathfrak{U} \subset \mathfrak{U}^{d} \subset \mathfrak{F}
$$

and by the previous results,

$$
G=\operatorname{Aut}_{\mathfrak{Q}^{d}} \mathfrak{\Im} .
$$

The full gauge group $\Gamma$ can now be defined by

$$
\Gamma=\operatorname{Aut}_{\mathfrak{N}} \mathfrak{E} \text {. }
$$

As shown in [3, Sect. 2], $\Gamma$ acts locally on the field net:

$$
\gamma(\mathfrak{F}(\mathcal{O}))=\mathfrak{F}(\mathcal{O}), \quad \gamma \in \Gamma
$$

We impose the physically reasonable requirement that the local energy-momentum tensor be an observable (cf. [3, Sect. 2]), so that $\Gamma$ commutes with the spacetime translations on $\mathfrak{F}$ and the vacuum state $\tilde{\omega}_{0}$ of $\mathfrak{F}$ is transformed by 
$\gamma \in \Gamma$ into a vacuum state $\tilde{\omega}_{0} \circ \gamma$ on $\mathfrak{F}$ which is also an extension of the vacuum state $\omega_{0}$ on $\mathfrak{A}$. The reference vacuum state $\tilde{\omega}_{0}$ is the unique $G$-invariant vacuum state of $\mathfrak{F}$ which is normal on $\mathfrak{P}^{d}$.

The natural question here is whether $\tilde{\omega}_{0} \circ \gamma, \gamma \in \Gamma$ exhausts the set of pure vacuum state extensions of $\omega_{0}$ to $\mathfrak{F}$. We are only able to offer a partial positive answer to this question by characterizing those extensions of $\omega_{0}$ which lie on the orbit of $\tilde{\omega}_{0}$ under the normalizer of $G$ in $\Gamma$. To this end, we will study the set $\mathscr{V}$ of vacuum states of $\mathfrak{F}$ defined as follows: a state $\omega$ of $\mathfrak{F}$ is in $\mathscr{V}$ if and only if
a) $\omega \mid \mathfrak{U}=\omega_{0}$,
b) $\omega$ is translation invariant,
c) $\omega$ is $G$-invariant,
d) $\pi\left(\mathfrak{H}\left(\mathcal{O}^{\prime}\right)\right)^{\prime}=\pi\left(\mathfrak{Q}^{d}(\mathcal{O})\right)$, for each double cone $\mathcal{O}$,

where $\pi$ denotes the GNS representation obtained from the restriction of $\omega$ to $\mathfrak{I}^{d}$. We express d) by saying that $\omega$ preserves essential duality since the condition can also be written as an equality of nets:

$$
\pi \circ \mathfrak{Q}^{d}=(\pi \circ \mathfrak{Q})^{d} .
$$

Restricting the family $\mathscr{V}$ of states on $\mathfrak{F}$ to $\mathfrak{A}^{d}$, we get a family $\mathscr{W}$ of states on $\mathfrak{Q}^{d}$. $\mathscr{W}$ determines $\mathscr{V}$ completely since any $G$-invariant state $\omega$ on $\mathfrak{F}$ satisfies

$$
\omega=\omega \mid \mathfrak{X}^{d} \circ m,
$$

where $m$ denotes the (normal, completely positive) map of $\mathscr{B}(\mathscr{H})$ onto $U(G)^{\prime}$ got by integrating $\operatorname{Ad} U(g)$ with respect to the normalized Haar measure on $G$. Here $U(g)$ is the unitary implementing $g \in G$ on the Hilbert space of the GNS representation of the $G$-invariant state $\tilde{\omega}_{0}$.

Let $H \subset$ Aut $\mathfrak{Q}^{d}$ be the group of all automorphisms $\beta$ of the $C^{*}$-algebra $\mathfrak{U}^{d}$ which leave $\mathfrak{A}$ pointwise fixed. By our standing assumptions they automatically commute with translations. It follows immediately from the definition of $\beta$ and the dual net that

$$
\beta\left(\mathfrak{A}^{d}(\mathcal{O})\right)=\mathfrak{U}^{d}(\mathcal{O}), \quad \beta \in H, \quad \mathcal{O} \in \mathscr{K} .
$$

Given $\omega \in \mathscr{W}$, let $\left(\pi_{\omega}, \mathscr{U}_{\omega}\right)$ denote the covariant representation of $\left\{\mathfrak{U}^{d}, \alpha\right\}$ arising from the GNS-construction.

Proposition 4.1. The map $\beta \in H \mapsto \omega_{0} \circ \beta$ is a $1-1$ correspondence of $H$ onto $\mathscr{W}$; if $\omega=\omega_{0} \circ \beta, \beta \in H$, then

$$
\left(\pi_{\omega}, \mathscr{U}_{\omega}\right) \simeq\left(\pi_{0} \circ \beta, \mathscr{U}_{0}\right)
$$

so that each $\omega \in \mathscr{W}$ is locally normal, fulfills the spectrum condition and thus is indeed a vacuum state.

Proof. If $\beta \in H, \omega_{0} \circ \beta$ is in $\mathscr{W}$ and $\left(\pi_{0} \circ \beta, \mathscr{U}_{0}\right)$ is covariant since $\alpha_{a} \beta=\beta \alpha_{a}$, $a \in \mathbb{R}^{4}$, so that (4.7) follows easily. Furthermore, $\beta$ is uniquely determined by $\omega$ since, for $A, A^{\prime} \in \mathfrak{A}, B \in \mathfrak{U}^{d}, \omega\left(A B A^{\prime}\right)=\omega_{0} \beta\left(A B A^{\prime}\right)=\omega_{0}\left(A \beta(B) A^{\prime}\right)$ so that $\omega_{0} \circ \beta$ $=\omega_{0} \circ \beta^{\prime}$ implies $\beta=\beta^{\prime}$. So if $\omega=\omega_{0} \circ \beta$ with $\beta$ an automorphism of $\mathfrak{U}^{d}$ leaving $\mathfrak{A}$ pointwise fixed and $\omega \in \mathscr{W}$, then $\omega=\omega_{0} \circ \alpha_{a} \beta \alpha_{a}^{-1}, a \in \mathbb{R}^{4}$, hence $\beta=\alpha_{a} \beta \alpha_{a}^{-1}$ and $\beta$ is actually in $H$. Thus we need only show that each $\omega \in \mathscr{W}$ is of the form $\omega_{0} \circ \beta$ with $\beta \in$ Aut $\mathfrak{I}^{d}$ leaving $\mathfrak{A}$ pointwise fixed. Note, first, that $\pi_{\omega} \mid \mathfrak{A}$ is irreducible and $\pi_{\omega}$ is locally normal. For by (4.4),

$$
\pi_{\omega}(\mathfrak{U})^{\prime} \subset \pi_{\omega}\left(\mathfrak{H}\left(\mathcal{O}^{\prime}\right)\right)^{\prime}=\pi_{\omega}\left(\mathfrak{U}^{d}(\mathcal{O})\right), \quad \mathcal{O} \in \mathscr{K},
$$


hence $\pi_{\omega}(\mathfrak{l})^{\prime} \subset$ Centre $\pi_{\omega}\left(\mathfrak{U}^{d}\right)=\mathbb{C} I$. Now, by assumption, $\pi_{\omega}\left(\mathfrak{U}^{d}(\mathcal{O})\right)$ is a von Neumann algebra and $\mathfrak{O}^{d}$ is a simple $C^{*}$-algebra [11] hence $\pi_{\omega}$ is locally an isomorphism and hence $\pi_{\omega}$ is locally normal. Since $\mathfrak{U}$ is irreducible, $\Omega$ is cyclic for $\mathfrak{A}$, hence by a) we can define a unitary operator $V$, intertwining the restrictions of $\pi_{\omega}$ and $\pi_{0}$ to $\mathfrak{A}$, by

$$
V \pi_{\omega}(A) \xi_{\omega}=\pi_{0}(A) \Omega, \quad A \in \mathfrak{A},
$$

so that, in particular, $V \pi_{\omega}\left(\mathfrak{H}\left(\mathcal{O}^{\prime}\right)\right) V^{-1}=\pi_{0}\left(\mathfrak{A}\left(\mathcal{O}^{\prime}\right)\right)$; and, taking commutants and using $\mathrm{c}$ ), we get

so that

$$
V \pi_{\omega}\left(\mathfrak{H}^{d}(\mathcal{O})\right) V^{-1}=\pi_{0}\left(\mathfrak{H}^{d}(\mathcal{O})\right)
$$

$$
\beta(B):=V \pi_{\omega}(B) V^{-1}, \quad B \in \mathfrak{A}^{d},
$$

defines an automorphism $\beta$ of $\mathfrak{U}^{d}$ fixing $\mathfrak{A}$ pointwise and such that $\omega=\omega_{0} \circ \beta$. By the above comments, such a $\beta$ is unique, commutes with $\alpha_{a}$ and is hence in $H$.

By (4.5) and Proposition 4.1, we can conclude that each state $\omega$ in $\mathscr{V}$ is locally normal on $\mathfrak{F}$ and that the associated covariant representation $\left(\pi_{\omega}, \mathscr{U}_{\omega}\right)$ of $\{\mathfrak{F}, \tilde{\alpha}\}$ fulfills the spectrum condition with $\pi_{\omega}$ being irreducible [1, Proposition 6.4].

Let $K$ denote the normalizer of $G$ in $\Gamma$. Since $\Gamma$ commutes with translations, each state $\tilde{\omega}_{0} \circ \gamma, \gamma \in K$, is translation invariant and $G$-invariant. We can now characterize the orbit of $\tilde{\omega}_{0}$ under $K$ as follows:

Theorem 4.2. Under our general assumptions implying that $\Gamma$ commutes with translations, the orbit of the reference vacuum $\tilde{\omega}_{0}$ under the normalizer of $G$ in $\Gamma$ coincides with the set $\mathscr{V}$ of degenerate vacua which preserve essential duality. We have a short exact sequence of groups

$$
1 \rightarrow G \rightarrow K \rightarrow H \rightarrow 1,
$$

where the second arrow is inclusion and the third is restriction to $\mathfrak{Q}^{d}$.

Proof. Since each $\tilde{\beta} \in K$ normalizes $G$ and $\tilde{\omega}_{0}$ is $G$-invariant, $\tilde{\omega}_{0} \circ \widetilde{\beta}$ is $G$-invariant, hence $\tilde{\omega}_{0} \circ \widetilde{\beta}=\tilde{\omega}_{0} \circ \widetilde{\beta} \circ m=\omega_{0} \circ \beta \circ m=\omega \circ m=\tilde{\omega}$. Now $\omega=\omega_{0} \circ \beta$ spans $\mathscr{W}$ by Proposition 4.1, so $\tilde{\omega}_{0} \circ \widetilde{\beta}$ spans $\mathscr{V}$ as $\widetilde{\beta}$ varies in $K$. The other assertions follow immediately from Proposition 3.1 and Corollary 3.2.

Remarks. We close with a few remarks on the more general case where local energy-momentum operators are not assumed to exist in $\mathfrak{U}$ so that $\Gamma$ might not commute with translations. The conclusions in Theorem 4.2 can still be drawn by Corollary 3.2 provided we assume that the centre of $G$ is discrete: let $H$ denote the local automorphisms of $\mathfrak{A}^{d}$ which leave $\mathfrak{A}$ pointwise fixed and commute with translations and let $K$ be its inverse image in the normalizer of $G$ in $\Gamma$ under the quotient map. Then by Corollary $3.2, K$ commutes with translations and the conclusions of Theorem 4.2 still hold.

\section{References}

1. Doplicher, S., Roberts, J.E.: Endomorphisms of $C^{*}$-algebras, cross products and duality for compact groups. Ann. Math. 130, 75-119 (1989)

2. Doplicher, S., Roberts, J.E.: Why there is a field algebra with a compact gauge group describing the superselection structure in particle physics. Commun. Math. Phys. 131, 51-107 (1990) 
3. Buchholz, D., Doplicher, S., Longo, R., Roberts, J.E.: A new look at Goldstone's theorem. Reviews in Math. Phys. (to appear)

4. Doplicher, S., Roberts, J.E.: Compact group actions on $C^{*}$-algebras. J. Operator Theory 19, 283-305 (1988)

5. Haagerup, U., Størmer, E.: Equivalence of normal states on von Neumann algebras and the flow of weights. Adv. in Math. 83, 180-262 (1990)

6. Takesaki, M.: Duality for crossed products and the structure of von Neumann algebras of type III. Acta Math. 131, 249-310 (1979)

7. Guido, D., Longo, R.: Relativistic invariance and charge conjugation in quantum field theory. Commun. Math. Phys. (to appear)

8. Araki, H., Haag, R., Kastler, D., Takesaki, M.: Extensions of KMS states and chemical potential. Commun. Math. Phys. 53, 97-134 (1977)

9. Doplicher, S., Haag, R., Roberts, J.E.: Local observables and particle statistics. I. Commun. Math. Phys. 23, 199-230 (1971); II. Commun. Math. Phys. 35, 49-85 (1974)

10. Doplicher, S., Roberts, J.E.: A new duality theory for compact groups. Invent. Math. 98, 157-218 (1989)

11. Borchers, H.J.: A remark on a theorem of Misra. Commun. Math. Phys. 4, 315-323 (1967)

Communicated by A. Jaffe 